Pacific Journal of Mathematics

INFINITELY DIVISIBLE PROBABILITIES ON THE 


\title{
INFINITELY DIVISIBLE PROBABILITIES ON THE HYPERBOLIC PLANE
}

\author{
R. K. Getoor
}

1. Introduction. This paper may be regarded from two points of view. First of all it presents the theory of infinitely divisible radially symmetric probability measures on the hyperbolic plane and the naturally associated limit theorems. This point of view provided the motivation for the present paper and is explained in some detail in $\S 2$ and 3 . However, just as the analagous theory in the Euclidean case may be viewed as a chapter in the theory of Fourier transforms, so may the present theory be viewed as a chapter in the theory of Legendre transforms. That is, by using the harmonic analysis described in $\S \S 2$ and 3 one can set up a one-to-one correspondence between the radially symmetric probability measures, $\mu$, on the hyperbolic plane and certain functions of a complex variable $\phi(z)$ in such a way that the convolution of $\mu_{1}$ and $\mu_{2}$ on the hyperbolic plane corresponds to the pointwise product of their "transforms" $\phi_{1}$ and $\phi_{2}$. Since $\mu$ is radially symmetric it is completely specified by a distribution function $F(\lambda)$ on $\lambda \geqq 0$ and the correspondence between $\phi$ and $\mu$ (or $F$ ) is given by

$$
\phi(z)=\int_{0}^{\infty} K(z, \lambda) d F(\lambda)
$$

where $K(z, \lambda)$ is a certain Legendre function given by (4.9). The convolution of $\mu_{1}$ and $\mu_{2}$, at least in the case where $F_{1}$ and $F_{2}$ have densities, is written down explicitly in (3.9).

This second point of view is adopted for the most part beginning in $\S 4$ and so the majority of the paper (sections 4-10) deals with certain problems in the theory of the Legendre transform (1.1). The tools we use are those of classical analysis, but the problems treated are motivated by probability theory. The main results of the paper are contained in $\S \S 7$ and 8. In $\S 10$ Gaussian and stable distributions are defined within the present context. Finally in $\S 11$ we indicate the extensions of these ideas to a wider class of Legendre transforms which includes the theory of radially symmetric probability measures on the higher dimensional hyperbolic spaces as special cases.

We would like to thank Professor H. P. McKean who first introduced us to the material in $\S 2$, and who expressed interest and encouragement when the present paper was in its formative stages.

Received January 10, 1961. This research was supported, in part, by the National Science Foundation. 
2. General Remarks. The present section is devoted to a general situation that we will specialize to the hyperbolic plane in the next section. We follow, more or less, the expository article of Godement [4]. Let $G$ be a locally compact second countable topological group and let $K$ be a compact subgroup. Let $x, y, z$ denote elements of $G$ and $u$, $v$ elements of $K$. We define two equivalence relations on $G$ as follows:

$$
\begin{gathered}
x \sim y \Longleftrightarrow x y^{-1} \in K \\
x \approx y \Longleftrightarrow \text { there exist } u, v \in K \text { such that } x=u y v .
\end{gathered}
$$

Thus $H=G / \sim$ is the space of right cosets and $R=G / \approx$ is the space of double cosets. We give $H$ and $R$ the usual quotient space topology. Let $d x$ be right invariant Haar measure on $G$, then $d x$ induces measures $d h$ and $d r$ on $H$ and $R$ which are invariant under the (right) action of $G$. In order to avoid notational complications it will be convenient to regard all functions as being defined on $G$. Thus the statement $f \in L_{1}(R)$ will mean $f(x)=f(y)$ if $x \approx y$ and $\int|f(x)| d x<\infty$ with the obvious conventions for functions defined almost everywhere. Thus we have

$$
L_{p}(R) \subset L_{p}(H) \subset L_{p}(G)
$$

for each $p>0$.

If $f$ and $g$ are in $L_{1}(G)$ we define their convolution

$$
f * g .(x)=\int f\left(x y^{-1}\right) g(y) d y .
$$

It is well known that $L_{1}(G)$ is a Banach algebra and it is immediate that $L_{1}(R)$ and $L_{1}(H)$ are closed sub-algebras. The basic assumption of [4] is that $L_{1}(R)$ be commutative. Selberg [6] has shown that if $H$ is a symmetric (or more generally, weakly symmetric) space then $L_{1}(R)$ is commutative. For us the following simple sufficient condition (whose proof is a routine calculation and is therefore omitted) will suffice.

THEOREM 2.1. If $x \approx x^{-1}$ for all $x$ in $G$, then $L_{1}(R)$ is commutative.

In the remainder of this section we will assume that $L_{1}(R)$ is commutative. If $\alpha$ is a multiplicative linear functional on $L_{1}(R)$ then

$$
\alpha(f)=\int p_{\alpha}(x) f(x) d x
$$

where $p_{\alpha}$ is in $L_{\infty}(R)$. It is easy to see that $\alpha$ defined by (2.5) is a multiplicative linear functional if and only if

$$
p_{\alpha}(x) p_{\alpha}(y)=\int_{K} p_{\alpha}(x u y) d u
$$

\footnotetext{
1 This remark is due to $\mathrm{H}$. P. McKean.
} 
for almost all $x, y$. Here $d u$ is normalized Haar measure on $K$. Moreover (2.6) implies that $p_{a}$ is equal almost everywhere to a continuous function and thus the multiplicative linear functionals on $L_{1}(R)$ may be identified with the bounded continuous functions on $R$ satisfying (2.6). Such functions are called spherical functions on $R$.

Let $C_{\infty}$ be the continuous functions on $G$ with compact support. If $f \in C_{\infty}$ we define $\widetilde{f}(x)=\bar{f}\left(x^{-1}\right)$. A (signed) Radon measure $\mu$ on $G$ is said to be of positive type if

$$
\mu(\tilde{f} * f) \geqq 0
$$

for all $f \in C_{\infty}$. A continuous function $p$ on $G$ is of positive type if the measure $p(x) d x$ is. Let $\hat{R}$ be the totality of all spherical functions on $R$ which are of positive type. For $f \in L_{1}(R)$ we define

$$
\hat{f}(p)=\int f(x) p(x) d x \text { for } \quad p \in \hat{R},
$$

then, at least if $G$ is unimodular, one can develop a complete theory of harmonic analysis including a Plancherel theorem. For details see [4]. Since we won't need this general theory we will terminate our general discussion at this point.

3. The Hyperbolic Plane. Let $D$ be the interior of the unit disc in the complex plane, i.e., $D=\{z: z$ complex, $|z|<1\}$. The set $D$ furnished with the Riemannian metric

$$
d s^{2}=4\left(1-r^{2}\right)^{-2}\left[d x^{2}+d y^{2}\right]
$$

where $r^{2}=|z|^{2}=x^{2}+y^{2}$ will be called the hyperbolic plane. The geodesic joining $z_{1}$ and $z_{2}$ is the unique circle through them cutting the circle $|z|=1$ orthogonally. The hyperbolic distance $\zeta\left(z_{1}, z_{2}\right)$ between $z_{1}$ and $z_{2}$ is given by

$$
t h \zeta / 2=\left|z_{1}-z_{2}\right| \cdot\left|1-\bar{z}_{1} z_{2}\right|^{-1}
$$

where " $t h$ " denotes the hyperbolic tangent, similarly "ch" and "sh" will denote the hyperbolic cosine and sine. See [2] for a discussion of the hyperbolic plane including the above facts.

If $u$ and $b$ are complex numbers with $|u|=1$ and $|b|<1$ we define the hyperbolic motion $(u, b)$ as follows:

$$
(u, b): z \rightarrow u(z-b)(1-\bar{b} z)^{-1} .
$$

It is easy to check that (3.3) maps $D$ onto $D$ and preserves the hyperbolic distance (3.2). Let $G$ be the totality of all such motions with the obvious topology. Clearly $G$ is a topological group satisfying the hypotheses of $\S 2$. The multiplication in $G$ is composition, i.e., 


$$
\left(u_{1}, b_{1}\right)\left(u_{2}, b_{2}\right)=\left(\frac{u_{1}\left(u_{2}+b_{1} \bar{b}_{2}\right)}{u_{2}\left(\bar{u}_{2}+\bar{b}_{1} b_{2}\right)}, \frac{u_{2} b_{2}+b_{1}}{u_{2}+b_{1} \bar{b}_{2}}\right)
$$

and

$$
(u, b)^{-1}=(\bar{u},-u b) \text {. }
$$

Let $K$ be the compact subgroup consisting of motions $(u, 0)$, then $K$ is just the circle group. It is straight forward to check that the equivalence relations defined in (2.1) and (2.2) become

$$
\begin{gathered}
\left(u_{1}, b_{1}\right) \sim\left(u_{2}, b_{2}\right) \Longleftrightarrow b_{1}=b_{2} \\
\left(u_{1}, b_{1}\right) \approx\left(u_{2}, b_{2}\right) \Longleftrightarrow\left|b_{1}\right|=\left|b_{2}\right| .
\end{gathered}
$$

Thus $H$ can be identified with $D$ and $R$ with the half-open segment $0 \leqq r<1$. Moreover Haar measure in $G$ can be chosen so that the invariant measure induced in $H=D$ is just that associated with the Riemannian metric (3.1).

It is convenient to introduce geodesic polar coordinates $(\zeta, \theta)$ in $D$ with pole at $z=0$. Here $\zeta$ is the hyperbolic distance and the coordinates of the point $z=r e^{i \theta}$ with $0 \leqq \theta<2 \pi$ are $(\log 1+r / 1-r, \theta)$. In terms of these coordinates we have $d s^{2}=d \zeta^{2}+s h^{2} \zeta d \theta^{2}$ and the corresponding volume element is given by

$$
\operatorname{sh} \zeta \zeta d \theta \text {. }
$$

We now regard $R$ as the half-line $0 \leqq \zeta<\infty$ and write $f(\operatorname{ch} \zeta)$ for the generic function defined on $R$. Theorem 2.1, (3.5), and (3.7) imply that $L_{1}(R)$ is commutative and routine calculations show that if $f, g \in L_{1}(R)$, then their convolution is given by

$$
\begin{aligned}
f * g .(\operatorname{ch} \zeta) & =\int_{0}^{\infty} \int_{0}^{2 \pi} f(\operatorname{ch} \lambda) g[\operatorname{ch} \zeta \operatorname{ch} \lambda-\operatorname{sh} \zeta \operatorname{sh} \lambda \cos \theta] \operatorname{sh} \lambda d \theta d \lambda \\
& =\int_{0}^{\infty} \int_{0}^{2 \pi} f[\operatorname{ch} \zeta \operatorname{ch} \lambda-\operatorname{sh} \zeta \operatorname{sh} \lambda \cos \theta] g(\operatorname{ch} \lambda) \operatorname{sh} \lambda d \theta d \lambda .
\end{aligned}
$$

Moreover the defining equation (2.6) for spherical functions becomes

$$
p(\operatorname{ch} \lambda) p(\operatorname{ch} \zeta)=\frac{1}{2 \pi} \int_{0}^{2 \pi} p[\operatorname{ch} \lambda \operatorname{ch} \zeta-\operatorname{sh} \lambda \operatorname{sh} \zeta \cos \theta] d \theta .
$$

From (3.10) one can show that $p$ is a solution of the Legendre differential equation and since $p(1)=1$, it follows that the solutions of (3.10) are $P_{\nu}(c h \zeta)$ where $P_{\nu}$ denotes the Legendre function of the first kind. See [3]. Equation (3.10) is then a simple consequence of the usual addition theorem for Legendre functions [3].

Since the spherical functions are bounded we must have $-1 \leqq$ $R e(\nu) \leqq 0$. Finally it is not too difficult to see that $P_{\nu}(\operatorname{ch} \zeta)$ is of positive 
type if and only if $\operatorname{Re}(\nu)=-1 / 2$. Thus we have identified the spherical functions and the spherical functions of positive type for the hyperbolic plane. See also [4].

The fact that the two integrals in (3.9) are equal is, of course, a consequence of the general theory of $\S 2$. However, one can see this directly as follows. In the first integral in (3.9) we regard $(\lambda, \theta)$ as geodesic polar coordinates for the hyperbolic plane $D$ with pole at 0 . Let $z_{0}$ be the point whose coordinates are $(\zeta, 0)$ and let $(\mu, \varphi)$ be geodesic polar coordinates for $D$ with pole at $z_{0}$ with the same polar axis. Using (3.2) it is not difficult to see that relationship between the coordinates $(\lambda, \theta)$ and $(\mu, \varphi)$ of a point $z$ is

$$
\begin{aligned}
& \operatorname{ch} \mu=\operatorname{ch} \lambda \operatorname{ch} \zeta-\operatorname{sh} \lambda \operatorname{sh} \zeta \cos \theta \\
& \operatorname{ch} \lambda=\operatorname{ch} \mu \operatorname{ch} \zeta+\operatorname{sh} \mu \operatorname{sh} \zeta \cos \phi .
\end{aligned}
$$

Thus regarding the first integral in (3.9) as an integral over the hyperbolic plane we see that it is equal to (the volume elements are $\operatorname{sh} \lambda d \lambda d \theta$ and $\operatorname{sh} \mu d \mu d \varphi$, see (3.8))

$$
\int_{0}^{\infty} \int_{0}^{2 \pi} f[\operatorname{ch} \mu \operatorname{ch} \zeta+\operatorname{sh} \mu \operatorname{sh} \zeta \cos \varphi] g(\operatorname{ch} \mu) \operatorname{sh} \mu d \varphi d \mu
$$

and this is obviously equal to the second integral in (3.9). The relation expressed in (3.11) is just the law of cosines for hyperbolic trigonometry.

4. Preliminaries on Legendre Functions. We intend to study integral transforms of the form

$$
\varphi(\nu)=\int_{0}^{\infty} P_{\nu}(\operatorname{ch} \lambda) d F(\lambda)
$$

where $F$ is a bounded monotone nondecreasing function and $P_{\nu}(\operatorname{ch} \lambda)$ is the usual Legendre function. In this section we gather together some facts about the kernels $P_{\nu}(\operatorname{ch} \lambda)$ that we will need in the sequel.

Combining formula (3) of $\S 3.2$ and formula (22) of $\S 2.1$ of [3] we see that

$$
P_{\nu}(\operatorname{ch\lambda })=\left(\frac{1+\operatorname{ch} \lambda}{2}\right)^{\nu} F\left(-\nu,-\nu ; 1 ; \frac{\operatorname{ch} \lambda-1}{\operatorname{ch} \lambda+1}\right)
$$

for all complex $\nu$ provided $0 \leqq \lambda<\infty$, where $F$ is the usual hypergeometric function. In all statements to follow $\lambda$ is a nonnegative real number. It is immediate from (4.2) that for each fixed $\lambda$ the function $P_{\nu}(\operatorname{ch} \lambda)$ is an entire function of the complex variable $\nu$. Also

$$
\begin{gathered}
P_{\nu}(1)=1 \\
P_{0}(\operatorname{ch} \lambda)=P_{-1}(\operatorname{ch} \lambda)=1 .
\end{gathered}
$$


From formula (9) of $\S 3.7$ of [3] we have

$$
P_{\nu}(\operatorname{ch} \lambda)=-\frac{\sqrt{2}}{\pi} \sin \nu \pi \int_{0}^{\infty} \frac{\operatorname{ch}\left(\nu+\frac{1}{2}\right) t d t}{(\operatorname{ch} \lambda+\operatorname{cht})^{1 / 2}}
$$

provided $-1<\operatorname{Re}(\nu)<0$ and in particular for real $x$ we have

$$
P_{-\frac{1}{2}+i x}(\operatorname{ch} \lambda)=\frac{\sqrt{2}}{\pi} \operatorname{ch} \pi x \int_{0}^{\infty} \frac{\cos x t d t}{(\operatorname{ch} \lambda+\operatorname{ch} t)^{1 / 2}} .
$$

Using (4.2) above and the standard integral representation for the hypergeometric function ( $\$ 2.1 .3$ of [3]) we find that

$$
\begin{array}{r}
P_{\nu}(\operatorname{ch} \lambda)=\left(\frac{1+\operatorname{ch} \lambda}{2}\right)^{\nu}[\Gamma(-\nu) \Gamma(1+\nu)]^{-1} \\
\int_{0}^{1} t^{-\nu-1}(1-t)^{\nu}\left(1-\frac{\operatorname{ch} \lambda-1}{\operatorname{ch} \lambda+1} t\right)^{\nu} d t
\end{array}
$$

provided $-1<\operatorname{Re} \nu<0$. For $\nu$ real with $-1<\nu<0$ it is immediate from (4.6) that

$$
0 \leqq P_{\nu}(\operatorname{ch} \lambda) \leqq 1
$$

and since $P_{\nu}(\operatorname{ch} \lambda)$ is a continuous function of $\nu$ the inequality (4.7) must hold for all $\nu$ in the interval $-1 \leqq \nu \leqq 0$. On the other hand using formula (14) of $\S 3.7$ of [3] we have

$$
\left|P_{\nu}(\operatorname{ch} \lambda)\right| \leqq \frac{1}{\pi} \int_{0}^{\pi}[\operatorname{ch} \lambda+\operatorname{sh} \lambda \cos t]^{R e \nu} d t=P_{R e \nu}(\operatorname{ch} \lambda),
$$

and combining this with (4.7) we obtain

$$
\left|P_{\nu}(\operatorname{ch} \lambda)\right| \leqq 1
$$

provided $-1 \leqq R e \nu \leqq 0$.

Let $z=x+i y$ be a complex variable and define the function

$$
K(z, \lambda)=P_{-\frac{1}{2}+i z}(\operatorname{ch} \lambda)
$$

for $\lambda \geqq 0$ and $-1 / 2 \leqq y \leqq 1 / 2$. For each fixed $\lambda, K(z, \lambda)$ is an analytic function of $z$ in the strip $-1 / 2<y<1 / 2$ and is continuous in the closed strip $-1 / 2 \leqq y \leqq 1 / 2$. The properties (4.3) and (4.8) become

$$
\begin{gathered}
K(z, 0)=1, K\left(-\frac{i}{2}, \lambda\right)=K\left(\frac{i}{2}, \lambda\right)=1 \\
|K(z, \lambda)| \leqq 1
\end{gathered}
$$

Moreover $K(x, \lambda)$ is given by the right hand side of (4.5). 
5. Uniqueness and Continuity. Let $\mathscr{F}$ be the collection of all bounded monotone nondecreasing real valued functions defined on $0 \leqq$ $\lambda<\infty$ and normalized so that

$$
\begin{gathered}
F(0)=0 \\
F(\lambda) \rightarrow F(\mu) \text { as } \lambda \downarrow \mu \text { for all } \mu>0 .
\end{gathered}
$$

Note that $F(0+)$ need not be zero. Let $\mathscr{F}_{0}$ be those $F \in \mathscr{F}$ which satisfy

$$
F(\infty)=\lim _{\lambda \rightarrow \infty} F(\lambda)=1 .
$$

All integrals are to be in the Lebesgue-Stieltjes sense. Integrals over $0 \leqq \lambda<\infty$ will be written $\int_{0}^{\infty}$, while integrals over $0<\lambda<\infty$ will be written $\int_{0+}^{\infty}$.

It $\left\{F_{n}\right\}$ is a sequence in $\mathscr{F}$ and $F \in \mathscr{F}$, then we say that $F_{n}$ converges weakly to $F$ (written $F_{n} \rightarrow F$ ) provided

$$
\int_{0}^{\infty} f d F_{n} \rightarrow \int_{0}^{\infty} f d F
$$

for all continuous $f$ with compact support. We say that $F_{n}$ is Bernoulli convergent to $F$ provided (5.3) holds for all bounded continuous $f$. It is obvious that if each $F_{n} \in \mathscr{F}_{0}$ and $F_{n} \Rightarrow F$, then $F \in \mathscr{F}_{0 .} \quad\left(F_{n} \Rightarrow F\right.$ means $F_{n}$ is Bernoulli convergent to $F$.)

If $K(z, \lambda)$ is the kernel defined in (4.9) we define the transform of $F \in \mathscr{F}$ by

$$
\varphi(z)=\int_{0}^{\infty} K(z, \lambda) d F(\lambda) .
$$

It is immediate that $\varphi$ is bounded in absolute value and continuous in the strip $-1 / 2 \leqq y \leqq 1 / 2$, and is analytic in $-1 / 2<y<1 / 2$. In particular $\phi(x)$ is a real valued even function of $x$, and $F \in \mathscr{F}_{0}$ if and only if $\phi(-i / 2)=1$. Of course, the values of $\varphi$ on the real axis completely determine $\rho$ in the strip $-1 / 2 \leqq y \leqq 1 / 2$. We now show that $F$ is uniquely determined by $\varphi$.

THEOREM 5.1 If $\varphi$ is the transform of $F$, then $\mathcal{Q}(x)$ uniquely determines $F$.

Proof. It suffices to prove that if $F$ is of bounded variation (not necessarily monotone) and if the integral in (5.4) vanishes for all $x$ then $F$ is identically zero. Using the representation (4.5) for $K(x, \lambda)$ we have

$$
\int_{0}^{\infty} h(t) \cos x t d t=0 \text { for all } x
$$


where

$$
h(t)=H(\operatorname{cht})=\int_{0}^{\infty}[\operatorname{ch} \lambda+\operatorname{cht}]^{-\frac{1}{2}} d F(\lambda) .
$$

The interchange of order of integration is justified since

$$
|h(t)| \leqq \operatorname{Var}(F)[1+\operatorname{cht}]^{-\frac{1}{2}}
$$

Moreover $h$ is continuous and the above inequality implies $h \in L_{1}$, hence (5.5) coupled with the uniqueness theorem for Fourier integrals yields $h(t)=0$ for all $t \geqq 0$. Thus if we define $G(u)=F\left(c h^{-1}(u)\right)$ for $u \geqq 1$ and $G(u)=0$ for $0 \leqq u<1$ we have

$$
H(t)=\int_{0}^{\infty}(u+t)^{-\frac{1}{2}} d G(u)=0
$$

for all $t \geqq 1$. But

$$
\begin{aligned}
H(t) & =\pi^{-\frac{1}{2}} \int_{0}^{\infty} \int_{0}^{\infty} e^{-\sigma(u+t)} \sigma^{-\frac{1}{2}} d \sigma d G(u) \\
& =\pi^{-\frac{1}{2}} \int_{0}^{\infty} e^{-\sigma t} \sigma^{-\frac{1}{2}} g(\sigma) d \sigma
\end{aligned}
$$

where $g(\sigma)$ is the Laplace-Stieltjes transform of $G$. Since $H(t)=0$ for $t \geqq 1$ we see that $\sigma^{-\frac{1}{2}} g(\sigma)=0$ for almost all $\sigma$ which in turn implies that $G$, and hence $F$, is zero. Here we have used the uniqueness theorem [7] for Laplace transforms twice.

In the present work the following rather weak continuity theorem will suffice.

THEOREM 5.2. (i) If $F_{n} \rightarrow F$ and $F_{n}(\infty) \leqq M$ then $\varphi_{n}(x) \rightarrow \varphi(x)$.

(ii) Let $\varphi_{n}$ be the transform of $F_{n}$ and suppose $\varphi_{n}(-i / 2)=F_{n}(\infty) \leqq$ $M$, then if $\varphi_{n}(x) \rightarrow \varphi(x)$ there exists an $F \in \mathscr{F}$ such that $F_{n} \rightarrow F$ and $\varphi$ is the trasform of $F$.

Proof. (i) For each $x$ the function $K(x, \cdot)$ is continuous and (4.5) implies that it vanishes at infinity. Thus it is immediate that for each fixed $x$ we have $\varphi_{n}(x) \rightarrow \varphi(x)$.

(ii) Since $F_{n}(\infty) \leqq M$ the Helly theorem implies the existence of a subsequence $\left\{F_{n}^{\prime}\right\}$ weakly convergent to $F^{\prime}$. If $\phi^{\prime}$ is the transform of $F^{\prime}$ then (i) implies that $\phi^{\prime}(x)=\varphi(x)$ for all $x$. If the entire sequence $\left\{F_{n}\right\}$ does not converge to $F^{\prime}$, then there exists another subsequence $\left\{F_{n}^{\prime \prime}\right\}$ converging to $F^{\prime \prime} \neq F^{\prime}$. But as before $\varphi^{\prime \prime}(x)=\varphi(x)=\phi^{\prime}(x)$ which contradicts the uniqueness theorem. Thus if we let $F=F^{\prime \prime}$ the proof of (ii) is complete. 
REMARK 1. Since the limit $\varphi$ in (ii) above is a transform it follows that $\varphi(z)$ may be defined as a continuous function on the strip $-1 / 2 \leqq$ $y \leqq 1 / 2$ which is analytic in the open strip. If $\varphi(-i / 2)=\lim _{n \rightarrow \infty} \varphi_{n}(-i / 2)$, then $F_{n}(\infty) \rightarrow F(\infty)$ which implies that $F_{n} \Rightarrow F$.

REMARK 2. If $F_{n} \Rightarrow F$ then since $K(z, \lambda)$ is continuous and bounded for $\lambda \geqq 0$ and $z$ in the strip $-1 / 2 \leqq y \leqq 1 / 2$, it follows that $\varphi_{n}(z) \rightarrow \varphi(z)$ uniformly on each compact sub strip $-1 / 2 \leqq y \leqq 1 / 2$ and $0 \leqq x \leqq x_{0}$. See, for example, Lemma 1.5.2 (iv) of [1].

6. Closure Properties. Let $\mathscr{P}\left(\mathscr{P}_{0}\right)$ be the class of all transforms of functions in $\mathscr{F}\left(\mathscr{F}_{0}\right)$. It is then immediate that if $\varphi$ and $\psi$ are in $\mathscr{P}$ and $\alpha, \beta \geqq 0$ then $\alpha \varphi+\beta \psi \in \mathscr{P}$, while if $\varphi, \psi \in \mathscr{P}_{0}$ and $\alpha+\beta=1$, $\alpha \geqq 0, \beta \geqq 0$, then $\alpha \varphi+\beta \psi \in \mathscr{P}_{0}$. Moreover Theorem 5.2 (ii) implies that if $\left\{\varphi_{n}\right\}$ is a sequence in $\mathscr{P}_{0}$ and $\varphi_{n}(x) \rightarrow \varphi(x)$, then $\varphi \in \mathscr{P}$.

The main result of this section is that $\mathscr{P}$ and $\mathscr{P}$ are closed under pointwise products. The proof of the following theorem is, of course, motivated by the general discussion of $\S \S 2$ and 3 .

THEOREM 6.1. If $\varphi_{1}$ and $\varphi_{2}$ are in $\mathscr{P}(\mathscr{P})$ then $\varphi_{1} \varphi_{2}$ is in $\mathscr{P}(\mathscr{P})$.

Proof. Let $\varphi_{1}$ and $\varphi_{2}$ be the transforms of $F_{1}$ and $F_{2}$. We first consider the case in which $F_{1}$ and $F_{2}$ have continuous densities $f_{1}(\operatorname{ch} \lambda)$ and $f_{2}(\operatorname{ch} \lambda)$ with respect to the measure $\operatorname{sh} \lambda d \lambda$. Of course, $f_{j}(\operatorname{ch} \lambda)$ may be unbounded near $\lambda=0$. Thus

$$
\begin{array}{r}
\Phi_{j}(x)=\int_{0}^{\infty} K(x, \lambda) f_{j}(\operatorname{ch} \lambda) \operatorname{sh} \lambda d \lambda \quad j=1,2 \\
\int_{0}^{\infty} f_{j}(\operatorname{ch} \lambda) \operatorname{sh} \lambda d \lambda<\infty ; f_{\jmath}(\operatorname{ch} \lambda) \geqq 0 ; j=1,2 .
\end{array}
$$

For the purposes of the present proof it will be convenient to write

$$
p(z, \operatorname{ch} \lambda)=K(z, \lambda)=P_{-\frac{1}{2}+i z}(\operatorname{ch} \lambda) .
$$

An immediate consequence of the addition theorem for Legendre functions [3] is that

$$
p(z, \operatorname{ch} \lambda) p(z, \operatorname{ch} \mu)=\frac{1}{2 \pi} \int_{0}^{2 \pi} p(z, a(\theta)) d \theta
$$

where

$$
a(\theta)=\operatorname{ch} \lambda \operatorname{ch} \mu-\operatorname{sh} \lambda \operatorname{sh} \mu \cos \theta .
$$

Therefore 


$$
\varphi_{1}(z) \varphi_{2}(z)=\frac{1}{2 \pi} \int_{0}^{\infty} f_{1}(\operatorname{ch} \lambda) \operatorname{sh} \lambda d \lambda \int_{0}^{\infty} \int_{0}^{2 \pi} f_{2}(\operatorname{ch} \mu) p(z, a(\theta)) \operatorname{sh} \mu d \theta d \mu
$$

If in the inner integrals we make the change of variable described in the last paragraph of $\S 3$ we find (the manipulations are justified since $f_{2}(\operatorname{ch} \mu) \in L_{1}(\operatorname{sh} \mu d \mu)$ and $p$ is bounded)

$$
\varphi_{1}(z) \varphi_{2}(z)=\int_{0}^{\infty} p(z, \operatorname{ch} \mu) h(\operatorname{ch} \mu) \operatorname{sh} \mu d \mu
$$

where

$$
h(\operatorname{ch} \mu)=\frac{1}{2 \pi} \int_{0}^{\infty} \int_{0}^{2 \pi} f_{1}(\operatorname{ch} \lambda) f_{2}(a(\theta)) \operatorname{sh} \lambda d \theta d \lambda
$$

clearly $h \geqq 0$ and

$$
F_{1}(\infty) F_{2}(\infty)=\varphi_{1}\left(-\frac{i}{2}\right) \varphi_{2}\left(-\frac{i}{2}\right)=\int_{0}^{\infty} h(\operatorname{ch} \mu) \operatorname{sh} \mu d \mu .
$$

Thus $\varphi_{1} \varphi_{2}$ is in $\mathscr{P}$, and if $\varphi_{1}, \varphi_{2}$ are in $\mathscr{P}_{0}$, then $\varphi_{1} \varphi_{2}$ is in $\mathscr{R}_{0}$.

We now turn to the general case. Let $k(\lambda)=e^{-\lambda}$ if $\lambda \geqq 0$ and $k(\lambda)=0$ if $\lambda<0$, and put $k_{n}(\lambda)=n k(n \lambda)$. Defining $F_{1}(\lambda)$ and $F_{2}(\lambda)$ to be zero for $\lambda<0$, it is clear that

$$
{ }_{n} f_{i}(\lambda)=\int_{-\infty}^{\infty} k_{n}(\lambda-\mu) d F_{i}(\mu)=\int_{0}^{\lambda} k_{n}(\lambda-\mu) d F_{i}(\mu)
$$

are continuous functions of $\lambda \geqq 0$. Moreover if we define ${ }_{n} F_{i}(\lambda)=$ $\int_{0}^{\lambda} f_{i}(\mu) d \mu$, it follows that ${ }_{n} F_{i} \in \mathscr{F}$, and ${ }_{n} F_{i} \in \mathscr{F}_{0}$ if $F_{i} \in \mathscr{F}_{0}$. Here $i=$ 1,2. It is well known [1, Th. 1.5.3] that ${ }_{n} F_{i} \Rightarrow F_{i}$ as $n \rightarrow \infty$. If ${ }_{n} g_{i}(\operatorname{ch} \lambda)={ }_{n} f_{i}(\lambda)$ then ${ }_{n} g_{i}(\operatorname{ch} \lambda)(\operatorname{sh} \lambda)^{-1}$ is the density of ${ }_{n} F_{i}$ with respect to $\operatorname{sh} \lambda d \lambda$. Thus if ${ }_{n} \rho_{i}$ is the transform of ${ }_{n} F_{i}$, it follows from what we proved above that $\psi_{n}(z)={ }_{n} \varphi_{1}(z)_{n} \mathscr{P}_{2}(z)$ is in $\mathscr{P}$ (or $\left.\mathscr{P}_{0}\right)$. But by the second remark following theorem 5.2 we have ${ }_{n} \varphi_{i}(z) \rightarrow \varphi_{i}(z)$ everywhere on the strip $-1 / 2 \leqq y \leqq 1 / 2$. Thus $\varphi_{1}(z) \varphi_{2}(z)=\lim _{n \rightarrow \infty} \psi_{n}(z)$ is in $\mathscr{P}$, and

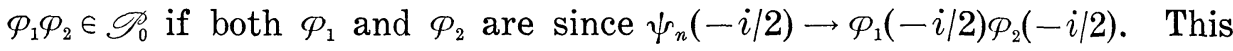
completes the proof of Theorem 6.1.

The following theorem gives another interesting closure property of $\mathscr{P}$.

TheOREM 6.2. If $\mathcal{P} \in \mathscr{P}$, then $\psi(z)=\exp (t[\mathcal{P}(z)-\phi(-i / 2)])$ is in $\mathscr{P}_{0}$ for all $t>0$.

Proof. If $\alpha=\varphi(-i / 2)=F(\infty) \geqq 0$, then

$$
\psi(z)=e^{-\alpha t} \lim _{n \rightarrow \infty} \sum_{k=0}^{n} \frac{t^{k} \varphi(z)^{k}}{k !} .
$$


Using Theorem 6.1, the fact that $\mathscr{P}$ is closed under positive linear combinations, and Theorem 5.2 it follows that $\psi \in \mathscr{P}$. (Theorem 5.2 is applicable since

$$
\sum_{k=0}^{n} \frac{t^{k} \mathcal{P}\left(-\frac{i}{2}\right)^{k}}{k !} \leqq e^{\alpha t}
$$

for all $n$.) Moreover $\psi(-i / 2)=1$ and so $\psi \in \mathscr{P}_{0}$.

7. Limit Theorems. In this section we will consider only the class $\mathscr{P}_{0}$. We begin by making the following definitions suggested by probability theory.

DEFINITION 1. $\varphi \in \mathscr{P}_{0}$ is infinitely divisible if for each positive integer $n$ there exists a $\psi_{n} \in \mathscr{P}_{0}$ such that $\varphi=\left(\psi_{n}\right)^{n}$.

Definition 2. $\varphi \in \mathscr{P}_{0}$ is a generalized limit if there exist $\varphi_{n k} \in \mathscr{P}_{n}$ for $n=1,2, \cdots$, and $k=1,2, \cdots, k_{n}$ with $k_{n} \rightarrow \infty$ as $n \rightarrow \infty$ such that

$$
\max _{k}\left|\varphi_{n k}(x)-1\right| \rightarrow 0
$$

uniformly on each bounded interval $0 \leqq x \leqq x_{0}<\infty$, and

$$
\varphi_{n}(x)=\prod_{k=1}^{k_{n}} \varphi_{n k}(x) \rightarrow \varphi(x)
$$

for all $x$.

In this section we will show that $\phi$ is infinitely divisible if and only if $\phi$ is a generalized limit, and at the same time obtain a canonical form for such $\varphi$. In the course of our discussion we will need the following two lemmas which we state here for convenience. The proofs of these lemmas will be given in $\S 9$.

LEMma 7.1. (i) There exist constants $\lambda_{0}>0$ and $M_{R}<\infty$ such that $\lambda^{-2}|1-K(z, \lambda)| \leqq M_{R}$ provided $0 \leqq \lambda \leqq \lambda_{0}$ and $|z| \leqq R$ (of course $z$ is in the strip $-1 / 2 \leqq y \leqq 1 / 2)$.

(ii) $\quad \lambda^{-2}[1-K(x, \lambda)] \rightarrow 1 / 4\left(1 / 4+x^{2}\right)$ as $\lambda \rightarrow 0$.

LEMMA 7.2. Let $H_{T}(\lambda)=\frac{1}{T} \int_{0}^{T}[1-K(x, \lambda)] d x$, then

(i) $0 \leqq H_{T} \leqq 2$ and $H_{T}(\lambda)>0$ for $\lambda>0$;

(ii) $H_{T}(\lambda) \rightarrow 1$ as $\lambda \rightarrow \infty$;

(iii) $\lambda^{-2} H_{T}(\lambda) \rightarrow C(T)>0$ as $\lambda \rightarrow 0$.

We begin with the simple half of our main result. 
THEOREM 7.1. If $\varphi$ is infinitely divisible, then $\varphi$ is a generalized limit.

Proof. Letting $k_{n}=n$ and $\varphi_{n k}=\psi_{n}$ for all $k$ we see that $\varphi_{n}$ (defined in (7.2)) is identical with $\varphi$ for all $n$. Thus we need only verify (7.1). But (4.10) implies that $|\varphi(x)| \leqq 1$, and, of course, $\varphi(x)$ and $\psi_{n}(x)$ are real. Therefore

$$
\psi_{n}(x)^{2}=\left[\varphi(x)^{2}\right]^{\frac{1}{n}} \rightarrow \psi(x)
$$

where $\psi(x)=0$ or 1 according as $\varphi(x)=0$ or $\varphi(x) \neq 0$. But $\varphi(0)>0$ and hence $\psi(0)=1$. Moreover $\psi_{n}^{2} \in \mathscr{P}_{0}$ and thus Theorem 5.2 implies that $\psi \in \mathscr{P}$. In particular $\psi(x)$ is continuous and since $\psi$ can only take on the values 0 and 1 it follows that $\psi(x)=1$ for all $x$. Hence $\varphi(x)$ never vanishes and since $\psi_{n}(x)=\exp [1 / n \log \varphi(x)]$ in a neighborhood (depending on $n$ ) of 0 we must have $\psi_{n}(x)=\exp [1 / n \log \varphi(x)]$ for all $x \geqq 0$. Therefore $\psi_{n}(x) \rightarrow 1$ as $n \rightarrow \infty$. This completes the proof of Theorem 7.1.

We now turn our attention to the converse of Theorem 7.1. This will not be established in full generality until $\S 8$. In working with Definition 2 we will adopt the convention that $F_{n k}$ is the element in $\mathscr{P}_{0}$ whose transform is $\varphi_{n k}$, similarly $\varphi_{n}$ is the transform of $F_{n}, \varphi$ of $F$. We begin with the following result.

THEOREM 7.2. Condition (7.1) of definition (2) is equivalent to

$$
\max _{k} \int_{\varepsilon}^{\infty} d F_{n k}(\lambda) \rightarrow 0
$$

as $n \rightarrow \infty$ for all $\varepsilon>0$.

Proof. Suppose (7.3) holds then

$$
\begin{aligned}
\max _{k} \mid \varphi_{n k}(x) & -1 \mid \leqq \max _{k} \int_{0}^{\varepsilon}[1-K(x, \lambda)] d F_{n k}(\lambda) \\
& +\max _{k} \int_{\varepsilon}^{\infty}[1-K(x, \lambda)] d F_{n k}(\lambda) \\
& \leqq \varepsilon^{2} M_{R}+2 \max _{k} \int_{\varepsilon}^{\infty} d F_{n k}(\lambda)
\end{aligned}
$$

provided $\varepsilon<\lambda_{0}$ and $0 \leqq x \leqq R$ where $\lambda_{0}$ and $M_{R}$ are defined in Lemma 7.1. Thus (7.1) follows.

Suppose (7.1) holds and (7.3) does not hold, then there exists an $\varepsilon>0$ and a subsequence $n$, such that

$$
\max _{k} \int_{\varepsilon}^{\infty} d F_{n g k} \geqq \eta>0 \text {. }
$$


Let $k_{j}$ be the value of $k$ at which the maximum in (7.4) is attained $\left(0 \leqq k_{j} \leqq k_{n_{j}}\right)$ and let $G_{j}=F_{n_{j} k_{j}}$. Let $\psi_{j}$, be the transform of $G_{j}$, then (7.1) implies that $\psi_{j}(x) \rightarrow 1$ which is the transform of $E$ (unit mass at the origin). Thus $G_{j} \rightarrow E$ weakly, but this is clearly a contradiction since $\int_{\varepsilon}^{\infty} d G_{\jmath} \geqq \eta>0$.

THEOREM 7.3. If $\varphi$ is generalized limit then $\varphi(x)=\exp [-\psi(x)]$ where

$$
\psi(x)=\int_{0}^{\infty}[1-K(x, \lambda)] \frac{1+\lambda^{2}}{\lambda^{2}} d \Omega(\lambda)
$$

with $\Omega \in \mathscr{F}$.

Proof. Theorem 6.1 implies that each $\varphi_{n} \in \mathscr{P}_{0}$. Hence $F_{n}(\infty)=1=$ $F(\infty)$ and since $\varphi_{n}(x) \rightarrow \varphi(x)$ we see that $F_{n} \rightarrow F$. Combining these facts yields $F_{n} \Rightarrow F$. Thus $\varphi_{n}(z) \rightarrow \varphi(z)$ uniformly on each strip $-1 / 2 \leqq$ $y \leqq 1 / 2$ and $0 \leqq x \leqq x_{0}$. (Remarks following Theorem 5.2). Also $\varphi(x)$ can not vanish near $x=0$ since $\varphi \in \mathscr{P}_{0}$. Let $x_{0}$ be the first zero of $\varphi$, then $\varphi_{n}(x) \rightarrow \varphi(x)$ uniformly on $0 \leqq x \leqq x_{0}$. Condition (7.1) implies that $\varphi_{n k}(x)$ doesn't vanish for $0 \leqq x \leqq x_{0}$ and all $k$ provided that $n$ is sufficiently large (how large depending only on $x_{0}$ ). Thus for $0 \leqq x<x_{0}$ and $n$ sufficiently large we can write

$$
-\log \varphi_{n k}(x)=-\log \left\{1-\int_{0}^{\infty}[1-K(x, \lambda)] d F_{n k}(\lambda)\right\} .
$$

Letting

$$
a_{n k}(x)=1-\varphi_{n k}(x)=\int_{0}^{\infty}[1-K(x, \lambda)] d F_{n k}(\lambda) \geqq 0,
$$

it follows from (7.1) that $\alpha_{n k}(x) \rightarrow 0$ uniformly on $0 \leqq x \leqq x_{0}$ uniformly in $k$ as $n \rightarrow \infty$. Hence

$$
-\log \varphi_{n}(x)=-\sum_{k=1}^{k_{n}} \log \varphi_{n k}(x)=\sum_{k=1}^{k_{n}} \sum_{j=1}^{\infty}(j)^{-1}\left(a_{n k}\right)^{j},
$$

and letting $n \rightarrow \infty$ we obtain

$$
-\log \varphi(x)=\lim _{n \rightarrow \infty}\left\{\sum_{k=1}^{k_{n}} a_{n k}+\sum_{k=1}^{k_{n}} \sum_{j=2}^{\infty}(j)^{-1}\left(a_{n k}\right)^{j}\right\}
$$

provided $0 \leqq x<x_{0}$.

Since all the terms involved are nonnegative we have for $0 \leqq x<x_{0}$

$$
0 \leqq \sum_{k=1}^{k_{n}} \sum_{j=2}^{\infty}(j)^{-1}\left(a_{n k}\right)^{j} \leqq \max \left(a_{n k}\right) \sum_{k=1}^{k_{n}} \sum_{j=1}^{\infty}(j)^{-1}\left(a_{n k}\right)^{j}
$$




$$
\rightarrow 0(-\log \varphi(x))=0
$$

as $n \rightarrow \infty$. If we define

$$
G_{n}(\lambda)=\sum_{k=1}^{k_{n}} F_{n k}(\lambda)
$$

and use (7.7) and (7.8) we obtain

$$
-\log \varphi(x)=\lim _{n \rightarrow \infty} \int_{0}^{\infty}\left[1-K(x, \lambda) d G_{n}(\lambda)\right.
$$

provided $0 \leqq x<x_{0}$.

We now investigate the behavior of the functions $G_{n}$ as $n \rightarrow \infty$. It is an immediate consequence of Lemma 7.2 that for each $T>0$ there exists a constant $A(T)>0$ such that

$$
\frac{1}{T} \int_{0}^{T}[1-K(x, \lambda)] d x \geqq A(T) \frac{\lambda^{2}}{1+\lambda^{2}}
$$

for all $\lambda \geqq 0$. Also from (7.6) and the definition of $G_{n}$ it is clear that

$$
-\log \varphi_{n}(x) \geqq \int_{0}^{\infty}[1-K(x, \lambda)] d G_{n}(\lambda)
$$

for $0 \leqq x<x_{0}$ and $n$ sufficiently large. Moreover since $\varphi_{n}(x) \rightarrow \varphi(x)$ uniformly on $0 \leqq x \leqq x_{0}$ and $\varphi(x)$ is continuous and bounded away from zero on $0 \leqq x \leqq 1 / 2 x_{0}$ it follows that $\log \varphi_{n}(x) \rightarrow \log \varphi(x)$ uniformly on $0 \leqq x \leqq 1 / 2 x_{0}$. Thus if $0<T<1 / 2 x_{0}$ and $n$ large enough we have

$$
\begin{aligned}
\int_{0}^{\infty} \frac{\lambda^{2}}{1+\lambda^{2}} d G_{n}(\lambda) & \leqq[T A(T)]^{-1} \int_{0}^{T}-\log \varphi_{n}(x) d x \\
& \rightarrow[T A(T)]^{-1} \int_{0}^{T}-\log \varphi(x) d x<\infty .
\end{aligned}
$$

Hence there exists a constant $M<\infty$ such that

$$
\int_{0}^{\infty} \frac{\lambda^{2}}{1+\lambda^{2}} d G_{n}(\lambda) \leqq M
$$

Next we will show that given $\varepsilon>0$ there exists an $R$ (independent of $n$ ) such that

$$
\int_{R}^{\infty} d G_{n}(\lambda) \leqq \varepsilon .
$$

To this end we first note that $\varphi_{n}(i y) \rightarrow \varphi(i y)$ uniformly an $-1 / 2 \leqq y \leqq 1 / 2$. Also (4.2) and (4.9) yield $0<K(i y, \lambda) \leqq 1$ for $\lambda \geqq 0$ and $-1 / 2 \leqq y \leqq 1 / 2$ with $K(-i / 2, \lambda)=K(i / 2, \lambda)=1$. Thus $\varphi_{n}(i y)$ and $\varphi(i y)$ are strictly positive on $-1 / 2 \leqq y \leqq 1 / 2$. Since $a_{n k}(i y)=1-\varphi_{n k}(i y)<1$ an argument similar to the one leading to (7.6) yields 


$$
-\log \varphi_{n}(i y) \geqq \int_{0}^{\infty}[1-K(i y, \lambda)] d G_{n}(\lambda)
$$

Let $\eta>0$ be given, then since $\varphi(-i / 2)=1$ we can choose $T$ such that $-1 / 2<T<1 / 2$ and

$$
\frac{-2}{2 T+1} \int_{-\frac{1}{2}}^{T} \log \varphi(i y) d y<\eta \text {. }
$$

Moreover (4.4) and (4.9) imply $K(i y, \lambda) \rightarrow 0$ as $\lambda \rightarrow \infty$ for each fixed $y$ with $-1 / 2<y<1 / 2$. Thus we can choose $R_{0}$ so that

$$
\frac{2}{2 T+1} \int_{-\frac{1}{2}}^{T}[1-K(i y, \lambda)] d y>1-\eta
$$

for all $\lambda \geqq R_{0}$. Since $\varphi_{n}(i y) \rightarrow \varphi(i y)$ uniformly on $-1 / 2 \leqq y \leqq T$ and $\varphi$ is bounded away from zero there we can choose $n_{0}$ so that for $n>n_{0}$

$$
\frac{-2}{2 T+1} \int_{-\frac{1}{2}}^{T} \log \varphi_{n}(i y) d y \leqq \frac{-2}{2 T+1} \int_{-\frac{1}{2}}^{T} \log \varphi(i y) d y+\eta \leqq 2 \eta .
$$

Thus for $n>n_{0}$ we have

$$
\begin{aligned}
\int_{R_{0}}^{\infty}(1-\eta) d G_{n}(\lambda) & \leqq \frac{2}{2 T+1} \int_{-\frac{1}{2}}^{T} \int_{0}^{\infty}[1-K(i y, \lambda)] d G_{n}(\lambda) d y \\
& \leqq \frac{-2}{2 T+1} \int_{-\frac{1}{2}}^{T} \log \varphi_{n}(i y) d y \leqq 2 \eta
\end{aligned}
$$

or $\int_{R_{0}}^{\infty} d G_{n} \leqq \frac{2 \eta}{1-\eta}$ if $n>n_{0}$. It is now evident that given $\varepsilon>0$ one can choose an $R$ so that (7.11) holds (each $G_{n}$ being monotone nondecreasing and bounded).

Define

$$
\Omega_{n}(\lambda)=\int_{0}^{\lambda} \frac{t^{2}}{1+t^{2}} d G_{n}(t)
$$

Then each $\Omega_{n}$ is in $\mathscr{F}$. Using (7.10), the Helly theorem implies the existence of a subsequence (call it $\Omega_{n}$ again) such that $\Omega_{n} \rightarrow \Omega$ with $\Omega \in \mathscr{F}$ and $\Omega(\infty) \leqq M$. Moreover from (7.11)

$$
\int_{R}^{\infty} d \Omega_{n}(\lambda) \leqq \int_{R}^{\infty} d G_{n}(\lambda) \leqq \varepsilon
$$

uniformly in $n$ for $R$ sufficiently large and this easily implies that $\Omega_{n} \Rightarrow$

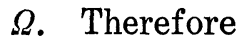

$$
\int_{0}^{\infty}[1-K(x, \lambda)] \frac{1+\lambda^{2}}{\lambda^{2}} d \Omega_{n}(\lambda) \rightarrow \int_{0}^{\infty}[1-K(x, \lambda)] \frac{1+\lambda^{2}}{\lambda^{2}} d \Omega(\lambda)
$$


where the integrand is defined by continuity, using Lemma 7.1, to be $1 / 4\left(1 / 4+x^{2}\right)$ at $\lambda=0$. Combining (7.9), (7.13), and (7.14) we obtain

$$
-\log \varphi(x)=\int_{0}^{\infty}[1-K(x, \lambda)] \frac{1+\lambda^{2}}{\lambda^{2}} d \Omega(\lambda)
$$

provided $0 \leqq x<x_{0}$. But $x_{0}$ was the smallest zero of $\varphi(x)$ and thus $-\log \varphi(x) \rightarrow \infty$ as $x \uparrow x_{0}$ while the integral in (7.15) remains bounded. Hence $\varphi(x)$ never vanishes and (7.15) must hold for all $x$. Finally defining $\psi(x)=-\log \varphi(x)$ we obtain Theorem 7.3.

THEOREM 7.4. A function $\psi$ has the representation (7.5) if and only if it can be written in the form

$$
\psi(x)=\frac{c}{4}\left(\frac{1}{4}+x^{2}\right)+\int_{0+}^{\infty}[1-K(x, \lambda)] d G(\lambda)
$$

where $c \geqq 0$ and $G$ is monotone nondecreasing, right continuous for $\lambda>0$, $G(\infty)=0$, and satisfying.

$$
\int_{0+}^{1} \lambda^{2} d G(\lambda)<\infty
$$

Proof. If $\psi$ has the representation (7.5) define $c=\Omega(0+) \geqq 0$ and $G(\lambda)=-\int_{\lambda+}^{\infty} t^{-2}\left(1+t^{2}\right) d \Omega(t)$ for $\lambda>0$, then using Lemma 7.1 (ii) it is clear that (7.16) holds and that $G$ has the required properties. Conversely if (7.16) hold define $\Omega(0)=0$ and $\Omega(\lambda)=c+\int_{0+}^{\lambda} t^{2}\left(1+t^{2}\right)^{-1} d G(t)$, then clearly $\Omega \in \mathscr{F}$ and (7.5) holds.

8. Uniqueness and Simple Consequences of (7.5). In order that our theory be reasonably complete the following uniqueness theorem is required.

THEOREM 8.1. The representation (7.5), and hence (7.16) also, is unique.

Proof. It is sufficient to prove that if $\Omega$ is of bounded variation and

$$
\psi(x)=\int_{0}^{\infty}[1-K(x, \lambda)] \frac{1+\lambda^{2}}{\lambda^{2}} d \Omega(\lambda)=0
$$

for all $x$, then $\psi=0$. We will use the following formula [5, p. 168] 


$$
(\operatorname{ch} \lambda+\operatorname{ch} \mu)^{-1}=\pi \int_{0}^{\infty} \frac{x \operatorname{sh} \pi x}{\operatorname{ch}^{2} \pi x} K(x, \lambda) K(x, \mu) d x
$$

which holds for all $\lambda, \mu \geqq 0$. (Robin's $K_{x}(\operatorname{ch} \lambda)$ is our $K(x, \lambda)$.) Since $K(0, \lambda)=1$, if we multiply (8.1) by $\pi x \operatorname{sh} \pi x(\operatorname{ch} \pi x)^{-2} K(x, \mu)$ and integrate from 0 to $\infty$ we obtain, using (8.2),

$$
\int_{0}^{\infty} \frac{\operatorname{ch} \lambda-1}{\operatorname{ch} \lambda+\operatorname{ch} \mu} \frac{1+\lambda^{2}}{\lambda^{2}} d \Omega(\lambda)=0 .
$$

The interchange of order of integration is justified since $(|K(x, \mu)| \leqq 1$ and $K(0, \mu)=1)$

$$
\begin{aligned}
\pi \int_{0}^{\infty} \frac{x \operatorname{sh} \pi x}{c h^{2} \pi x}|K(x, \mu)| & \int_{0}^{\infty}[1-K(x, \lambda)] \frac{1+\lambda^{2}}{\lambda^{2}} d|\Omega|(\lambda) d x \\
& \leqq \int_{0}^{\infty} \frac{\operatorname{ch} \lambda-1}{2(1+\operatorname{ch} \lambda)} \frac{1+\lambda^{2}}{\lambda^{2}} d|\Omega|(\lambda)<\infty,
\end{aligned}
$$

where $|\Omega|$ stands for the total variation of $\Omega$. But (8.3) may be written

$$
\int_{1}^{\infty}(t+s)^{-1} d \Omega^{*}(t)=0
$$

for all $s \geqq 1$, where for $t \geqq 1$

$$
\Omega^{*}(t)=\int_{0}^{c h^{-1}(t)} \lambda^{-2}\left(1+\lambda^{2}\right)(\operatorname{ch} \lambda-1) d \Omega(\lambda) .
$$

Noting that $\Omega^{*}$ is of bounded variation on each finite interval $1 \leqq t \leqq T$, we can apply the uniqueness theorem for Stieltjes transform [7, p. 336]. This leads to the conclusion that $\Omega^{*}$, and hence $\Omega$, is identically zero.

THEOREM 8.2. Given $a \psi$ of the form (7.5) then $\varphi=e^{-\psi}$ is an infinitely divisible element of $\mathscr{F}_{0}$.

Proof. Since $K(\cdot, \lambda)$ is in $\mathscr{P}$ for each $\lambda \geqq 0$, it follows from Theorem 6.2 that $\exp \{-b[1-K(\cdot, \lambda)]\}$ is in $\mathscr{P}$ for all $b, \lambda \geqq 0$. Thus if we approximate the integral defining $\psi(x)$ by a Riemann sum and use the above fact and Theorem 5.2, we find that $\varphi(x)=\exp [-\psi(x)]$ is in $\mathscr{P}$. Since $\varphi$ is in $\mathscr{P}$ it can be extended to a function $\varphi(z)$ which is continuous on the strip $-1 / 2 \leqq y \leqq 1 / 2$ and analytic on $-1 / 2<y<1 / 2$.

Using Lemma 7.1 (i) it is immediate that the integral in (7.5) converges for $z$ in the strip $-1 / 2 \leqq y \leqq 1 / 2$ to a function which we denote by $\psi(z)$. It is also clear that $\psi$ is continuous on $-1 / 2 \leqq y \leqq 1 / 2$ and analytic on $-1 / 2<y<1 / 2$. It now follows that $\varphi(z)=\exp [-\psi(z)]$ for all $z$ in the strip $-1 / 2 \leqq y \leqq 1 / 2$. Since $\psi(-i / 2)=0$ it follows that $\varphi \in \mathscr{P}_{0}$. Similarly $\exp [-1 / n \psi]$ is in $\mathscr{P}_{0}$ for each $n>0$ and thus $\varphi=$ $\exp (-\psi)$ is infinitely divisible. 
COROLlaRY 8.1. $\varphi$ is a generalized limit if and only if $\varphi$ is infinitely divisible.

Proof. This results from Theorems 7.1, 7.3, and 8.2.

Actually contained in the proofs of Theorem 7.3, 8.1, and 8.2 is the following result which we state explicitly for completeness.

THEOREM 8.3. Let $\varphi_{n K} \in \mathscr{P}_{0}$ for $n=1,2, \cdots$ and $k=1,2, \cdots, k_{n}$ with $k_{n} \rightarrow \infty$ and satisfy (7.1). Let $\varphi_{n}$ be defined by (7.2), then $a$ necessary and sufficient condition $\varphi_{n} \rightarrow \varphi \in \mathscr{P}_{0}$ is that

$$
\Omega_{n}(\lambda)=\sum_{k=1}^{k_{n}} \int_{0}^{\lambda} \frac{t^{2}}{1+t^{2}} d F_{n k}(t)
$$

be Bernoulli convergent to $\Omega \in \mathscr{F}$. In this case $\varphi=\exp (-\psi)$ where $\psi$ is defined by (7.5).

9. Proofs of the Lemmas. We begin with Lemma 7.1 (i). In view of the definition (4.9) of $K(z, \lambda)$ it will suffice to show $\lambda^{-2}\left|1-P_{\nu}(\operatorname{ch} \lambda)\right| \leqq$ $M_{r}$ for $0 \leqq \lambda \leqq \lambda_{0}$ and $|\nu| \leqq r$. Choose $\lambda_{0}$ such that the inequalities.

$$
\operatorname{ch} \lambda-1<1 ; \lambda^{-2}(\operatorname{ch} \lambda-1)<1
$$

hold for $0 \leqq \lambda \leqq \lambda_{0}$. Since $[3$, p. 122]

$$
P_{\nu}(\operatorname{ch} \lambda)=F\left(-\nu, \nu+1 ; 1 ; \frac{1}{2}(1-\operatorname{ch} \lambda)\right)
$$

provided $|1-\operatorname{ch} \lambda|<2$, we easily find that for $0 \leqq \lambda \leqq \lambda_{0}$

$$
\lambda^{-2}\left|1-P_{\nu}(\operatorname{ch} \lambda)\right| \leqq F\left(|\nu|,|\nu|+1 ; 1 ; \frac{1}{2}\right) .
$$

Taking $M_{r}=F(r, r+1 ; 1 ; 1 / 2)$ we obtain the desired conclusion.

Let $\lambda_{0}$ be as above, then from (9.2) and (4.9) we have for $0 \leqq \lambda \leqq \lambda_{0}$

$$
K(x, \lambda)=F\left[\frac{1}{2}-i x, \frac{1}{2}+i x ; 1 ; \frac{1}{2}(1-\operatorname{ch} \lambda)\right] .
$$

Expanding the hypergeometric function we find that

$$
\lambda^{-2}[1-K(x, \lambda)]+\frac{1}{2} \lambda^{-2}\left[\left(\frac{1}{4}+x^{2}\right)(1-\operatorname{ch} \lambda)\right]=G(x, \lambda)
$$

where $G(x, \lambda) \rightarrow 0$ as $\lambda \rightarrow 0$ for each fixed $x$. The second conclusion of Lemma 7.1 is now immediate.

Finally all of the conclusions of Lemma 7.2 with the possible exception of $H_{T}(\lambda)>0$ for $\lambda>0$ are easy consequences of Lemma 7.1 and 
(4.5). But if $\mathrm{H}_{T}\left(\lambda_{0}\right)=0$ for $\lambda_{0}>0$ we would have $K\left(x, \lambda_{0}\right)=1$ for all $x$ in $[0, T]$. In particular $K\left(0, \lambda_{0}\right)=1$. Using (4.5) and the fact that $K(0,0)=1$ this leads to the conclusion that

$$
\int_{0}^{\infty} \frac{d t}{\left[\operatorname{ch} \lambda_{0}+\operatorname{cht}\right]^{1 / 2}}=\int_{0}^{\infty} \frac{d t}{[1+\operatorname{ch} t]^{1 / 2}}
$$

which is clearly impossible if $\lambda_{0}>0$. This then completes the proofs of our lemmas.

10. Gaussian and Stable Distributions. It is an immediate consequence of Theorems 8.2 and 7.4 that for each $t>0$ the function

$$
\varphi(x)=\exp \left[-t\left(\frac{1}{4}+x^{2}\right)\right]
$$

is an infinitely divisible element of $\mathscr{P}_{0}$. Following Bochner [1] a $\varphi$ of the form (10.1) will be called Gaussian (or normal). Let $U_{t}(\lambda)$ be the element in $\mathscr{F}_{0}$ corresponding to $\exp \left[-t\left(1 / 4+x^{2}\right)\right]$. If one uses the inversion formula of Fock [5, p. 165] and the fact that [5, p. 154]

$$
K(x, \lambda)=\frac{\sqrt{2}}{\pi} \operatorname{coth} \pi x \int_{\lambda}^{\infty} \frac{\sin x t d t}{(\operatorname{cht}-\operatorname{ch} \lambda)^{1 / 2}}
$$

one finds that

$$
U_{t}(\lambda)=\int_{0}^{\lambda} u(t, \mu) \operatorname{sh} \mu d \mu
$$

where

$$
u(t, \lambda)=\frac{e^{-t / 4}}{(2 t)^{3 / 2}} \int_{\lambda}^{\infty} \frac{s e^{-s^{2} / 4 t} d s}{(\operatorname{chs}-\operatorname{ch} \lambda)^{1 / 2}} .
$$

The function $u(t, \lambda)$ defined in (10.2) is therefore the density (with respect to $\operatorname{sh} \lambda d \lambda$ ) of the rotationally invariant Gaussian distribution on the hyperbolic plane. It is not difficult to check directly that

$$
\int_{0}^{\infty} u(t, \lambda) \operatorname{sh} \lambda d \lambda=1 \text { for all } t>0,
$$

although it is not necessary for us to do so since we know that $U_{t} \in \mathscr{F}_{0}$. Finally it is interesting, but not unexpected, to note that

$$
\frac{\partial u}{\partial t}=(\operatorname{sh} \lambda)^{-1} \frac{\partial}{\partial \lambda}\left(\operatorname{sh} \lambda \frac{\partial u}{\partial \lambda}\right)
$$

The differential operator on the right side of (10.3) is the radial part of the Laplace-Beltrami operator in geodesic polar coordinates $(\lambda, \theta)$ for the hyperbolic plane. 
Let $0<\alpha<1$ and let $g_{\alpha}(u)$ be the positive continuous function defined on $u \geqq 0$ by the relation

$$
e^{-s^{x}}=\int_{0}^{\infty} e^{-s u} g_{\alpha}(u) d u
$$

for $s \geqq 0$. See $\S 4.3$ of [1]. (The function $g_{\alpha}$ is the density of a onesided stable law of index $\alpha$ on the real line.) For $t>0$ we define

$$
u_{\alpha}(t, \lambda)=\int_{0}^{\infty} u\left(t^{\frac{1}{\alpha}} s, \lambda\right) g_{\alpha}(s) d s
$$

where $u(t, \lambda)$ is the normal density $(10.2)$. Clearly $u_{\alpha}(t, \cdot)$ is a probability density with respect to $s h \lambda d \lambda$. Moreover

$$
\int_{0}^{\infty} K(x, \lambda) u_{\alpha}(t, \lambda) \operatorname{sh}(\lambda) d \lambda=\exp \left[-t\left(1 / 4+x^{2}\right)^{\alpha}\right]
$$

Thus for each $t>0$ the function $\exp \left[-t\left(1 / 4+x^{2}\right)^{\alpha}\right]$ is an infinitely divisible element of $\mathscr{P}_{0}$. By analogy with the Euclidean case one might call the densities (10.5) or the transforms (10.6) stable. We will investigate the properties of these distributions in a future paper, in particular, we will give a fuller justification of the name stable.

11. Higher Dimensional Hyperbolic Spaces. All that has gone before can be easily extended to a more general class of integral transforms that are related to the higher dimensional hyperbolic spaces. Let $\mu$ be a real parameter with $\mu>-1 / 2$ and define the kernels

$$
\begin{gathered}
K_{\mu}(x, \lambda)=2^{\mu} \Gamma(\mu+1)(\operatorname{sh} \lambda)^{-\mu} P_{-1 / 2+i x}^{(\mu)}(\operatorname{ch} \lambda) \\
=\left(\frac{1+\operatorname{ch} \lambda}{2}\right)^{-\mu} F\left(1 / 2-i x, 1 / 2+i x ; \mu+1 ; \frac{1-\operatorname{ch} \lambda}{2}\right),
\end{gathered}
$$

where $P_{\nu}^{(\mu)}$ is the usual associated Legendre function [3]. Similarly we define $K_{\mu}(z, \lambda)$ for complex $z$ by replacing $x$ by $z$ in (11.1). Clearly $K_{\mu}(z, \lambda)$ is analytic in $z$ and it is not difficult to verify that

$$
\left|K_{\mu}(z, \lambda)\right| \leqq 1 \text { if }-\mu-1 / 2 \leqq y \leqq 1 / 2
$$

and

$$
K_{\mu}(z, 0)=1, \quad K_{\mu}(-i(\mu+1 / 2), \lambda)=1 .
$$

Moreover it follows from 3.7(8) of [3] that

$$
K_{\mu}(x, \lambda)=\sqrt{\frac{2}{\pi}} \frac{2^{\mu} \Gamma(\mu+1)}{\Gamma(\mu+1 / 2)(\operatorname{sh} \lambda)^{2 \mu}} \int_{0}^{\lambda}(\operatorname{ch} \lambda-\operatorname{cht})^{\mu-1 / 2} \cos x t d t,
$$

and combining this with (11.2) results in 


$$
-1 \leqq K_{\mu}(x, \lambda) \leqq 1
$$

If $\mu=0$ then $K_{\mu}$ reduces to the kernel $K$ considered in the previous sections.

Let $\mu=N / 2-1$ where $N \geqq 2$ is an integer, then the functions $K_{\mu}(z, \cdot)$ are the spherical functions on $N$-dimensional hyperbolic space and the functions $K_{\mu}(x, \cdot)$ are the spherical functions of positive type.

If for arbitrary $\mu>-1 / 2$ we define the $\mu$-transform of an element $F \in \mathscr{F}$ by

$$
\varphi(x)=\int_{0}^{\infty} K_{\mu}(x, \lambda) d F(\lambda)
$$

then all of the results of the preceding sections can be carried over to $\mu$-transforms with only minor changes. In particular, in (7.16) one must replace $c / 4\left(1 / 4+x^{2}\right)$ by $c /[4(\mu+1)]\left[(\mu+1 / 2)^{2}+x^{2}\right]$ and then the Gaussian elements have the form $\exp \left(-t\left[(\mu+1 / 2)^{2}+x^{2}\right]\right)$. The proofs require only minor technical changes with the exception of Theorem 6.1.

In order to prove the analog of Theorem 6.1 for $\mu$-transforms one needs the following formula

$$
K_{\mu}(x, \lambda) K_{\mu}(x, t)=\frac{2^{2 \mu} \Gamma(1+\mu)^{2}}{\pi \Gamma(2 \mu+1)} \int_{0}^{\pi} K_{\mu}(x, w) \sin ^{2 \mu} \theta d \theta
$$

where

$$
w=\operatorname{ch} \lambda \operatorname{ch} t+\operatorname{sh} \lambda \operatorname{sh} t \cos \theta .
$$

Formula (11.7) is a simple consequence of the addition theorem for associated Legendre functions (formula 80 of Peter Henrici, Addition Theorems for General Legendre and Gegenbauer Functions, Journ. of Rat. Mech. and Anal. (4) 1955; note the misprint in this formula, namely $-\mu-n$ should be $-\nu-n)$ and the orthogonality relations for Gegenbauer polynomials. Using (11.7) the fact that the product of two $\mu$-transforms is again a $\mu$-transform is proved by an argument similar to the one used in $\S 6$.

Note added in proof. Some results which are similar to a part of ours appeared in a paper by F. I. Karpelevitch, V. N. Tutubalin, and M. G. Šur entitled "Limit theorems for convolutions of distributions on Lobachevsky's plane and space", Theory of Probability and its Applications, 4, (1959), 432-436. These authors were particularly interested in convergence to the normal distribution.

\section{REFERENCES}

1. S. Bochner, Harmonic Analysis and the Theory of Probability, Berkeley and Los Angeles, 1955.

2. C. Caratheodory, Theory of Functions of a Complex Variable, Volume I, New York, 1954. 
3. A. Erdelyi, Higher Transcendental Functions, Volume I, Bateman Manuscript Project. New York, 1953

4. R. Godement, Introduction aux travaux de A. Selberg, Séminaire Bourbaki, No. 144 (1956-57).

5. L. Robin, Fonctions Sphériques de Legendre et Fonctions Spheriodales, Tome III. Paris, 1959.

6. A. Selberg, Harmonic analysis and discontinuous groups in weakly symmetric Riemannian spaces with applications to Dirichlet series, J. Indian Math. Soc., 20 (1956), 47-87.

7. D. V. Widder, The Laplace Transform, Princeton, 1946.

\section{Seattle Washington}




\section{PACIFIC JOURNAL OF MATHEMATICS}

\section{EDITORS}

\author{
RaLPh S. Phillips \\ Stanford University \\ Stanford, California \\ F. H. BRowNELL \\ University of Washington \\ Seattle 5 , Washington
}

A. L. Whiteman

University of Southern California

Los Angeles 7, California

L. J. Paige

University of California

Los Angeles 24, California

\author{
E. F. BECKENBACH \\ T. M. CHERRY
}

\author{
ASSOCIATE EDITORS

$\begin{array}{lll}\text { D. DERRY } & \text { H. L. ROYDEN } & \text { E. G. STRAUS } \\ \text { M. OHTSUKA } & \text { E. SPANIER } & \text { F. WOLF }\end{array}$

\section{SUPPORTING INSTITUTIONS}

\author{
UNIVERSITY OF BRITISH COLUMBIA \\ CALIFORNIA INSTITUTE OF TECHNOLOGY \\ UNIVERSITY OF CALIFORNIA \\ MONTANA STATE UNIVERSITY \\ UNIVERSITY OF NEVADA \\ NEW MEXICO STATE UNIVERSITY \\ OREGON STATE COLLEGE \\ UNIVERSITY OF OREGON \\ OSAKA UNIVERSITY \\ UNIVERSITY OF SOUTHERN CALIFORNIA
}

\author{
STANFORD UNIVERSITY \\ UNIVERSITY OF TOKYO \\ UNIVERSITY OF UTAH \\ WASHINGTON STATE COLLEGE \\ UNIVERSITY OF WASHINGTON \\ AMERICAN MATHEMATICAL SOCIETY \\ CALIFORNIA RESEARCH CORPORATION \\ HUGHES AIRCRAFT COMPANY \\ SPACE TECHNOLOGY LABORATORIES \\ NAVAL ORDNANCE TEST STATION
}

Mathematical papers intended for publication in the Pacific Journal of Mathematics should be typewritten (double spaced), and the author should keep a complete copy. Manuscripts may be sent to any one of the four editors. All other communications to the editors should be addressed to the managing editor, L. J. Paige at the University of California, Los Angeles 24, California.

50 reprints per author of each article are furnished free of charge; additional copies may be obtained at cost in multiples of 50 .

The Pacific Journal of Mathematics is published quarterly, in March, June, September, and December. The price per volume (4 numbers) is $\$ 12.00$; single issues, $\$ 3.50$. Back numbers are available. Special price to individual faculty members of supporting institutions and to individual members of the American Mathematical Society: $\$ 4.00$ per volume; single issues, $\$ 1.25$.

Subscriptions, orders for back numbers, and changes of address should be sent to Pacific Journal of Mathematics, 103 Highland Boulevard, Berkeley 8, California.

Printed at Kokusai Bunken Insatsusha (International Academic Printing Co., Ltd.), No. 6, 2-chome, Fujimi-cho, Chiyoda-ku, Tokyo, Japan.

\section{PUBLISHED BY PACIFIC JOURNAL OF MATHEMATICS, A NON-PROFIT CORPORATION}

The Supporting Institutions listed above contribute to the cost of publication of this Journal, but they are not owners or publishers and have no responsibility for its content or policies.

Reprinted 1966 in the United States of America 


\section{Pacific Journal of Mathematics}

\section{Vol. 11, No. 4}

A. V. Balakrishnan, Prediction theory for Markoff processes . . . . . . . . . . 1171

Dallas O. Banks, Upper bounds for the eigenvalues of some vibrating systems . . . . 1183

A. Białynicki-Birula, On the field of rational functions of algebraic groups ...... 1205

Thomas Andrew Brown, Simple paths on convex polyhedra .............. 1211

L. Carlitz, Some congruences for the Bell polynomials . . . . . . . . . . . . 1215

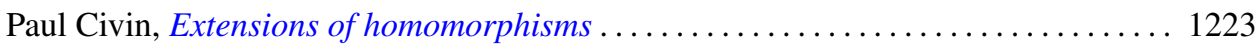

Paul Joseph Cohen and Milton Lees, Asymptotic decay of solutions of differential

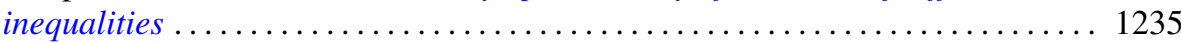

István Fáry, Self-intersection of a sphere on a complex quadric . . . . . . . . . . 1251

Walter Feit and John Griggs Thompson, Groups which have a faithful representation

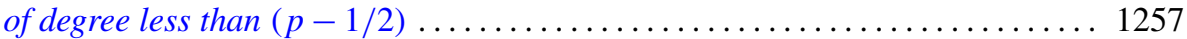

William James Firey, Mean cross-section measures of harmonic means of convex

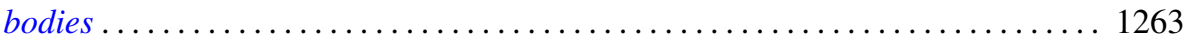

Avner Friedman, The wave equation for differential forms . . . . . . . . . . 1267

Bernard Russel Gelbaum and Jesus Gil De Lamadrid, Bases of tensor products of

Banach spaces ................................... 1281

Ronald Kay Getoor, Infinitely divisible probabilities on the hyperbolic plane . . . . 1287

Basil Gordon, Sequences in groups with distinct partial products . . . . . . . . . . . . 1309

Magnus R. Hestenes, Relative self-adjoint operators in Hilbert space . . . . . . . . . 1315

Fu Cheng Hsiang, On a theorem of Fejér ......................... 1359

John McCormick Irwin and Elbert A. Walker, On N-high subgroups of Abelian

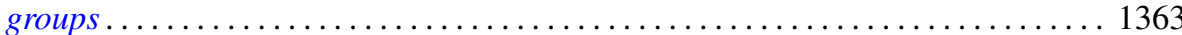

John McCormick Irwin, High subgroups of Abelian torsion groups . . . . . . . . . 1375

R. E. Johnson, Quotient rings of rings with zero singular ideal . . . . . . . . . . . 1385

David G. Kendall and John Leonard Mott, The asymptotic distribution of the time-to-escape for comets strongly bound to the solar system ...

Kurt Kreith, The spectrum of singular self-adjoint elliptic operators ....

Lionello Lombardi, The semicontinuity of the most general integral of the calculus of variations in non-parametric form ................................

Albert W. Marshall and Ingram Olkin, Game theoretic proof that Chebyshev inequalities are sharp

Wallace Smith Martindale, III, Primitive algebras with involution . . William H. Mills, Decomposition of holomorphs ..............

James Donald Monk, On the representation theory for cylindric algebras . . . . . . 1447

Shu-Teh Chen Moy, A note on generalizations of Shannon-McMillan theorem . . . . 1459

Donald Earl Myers, An imbedding space for Schwartz distributions . .

John R. Myhill, Category methods in recursion theory .........

Paul Adrian Nickel, On extremal properties for annular radial and circular slit mappings of bordered Riemann surfaces

Edward Scott O'Keefe, Primal clusters of two-element algebras . .

Nelson Onuchic, Applications of the topological method of Wazewski to certain

problems of asymptotic behavior in ordinary differential equations ...

Peter Perkins, A theorem on regular matrices................

Clinton M. Petty, Centroid surfaces .... 